\title{
Ultrasound and differential diagnosis of fetal abdominal cysts
}

\author{
CHANG-YU TU
}

\author{
Department of Ultrasound, Women and Children's Hospital of Linyi, Linyi, Shandong 276001, P.R. China
}

Received July 24, 2015; Accepted September 9, 2016

DOI: $10.3892 / \mathrm{etm} .2016 .3948$

\begin{abstract}
The present study aimed to investigate the use of ultrasound and differential diagnosis to diagnose a fetal abdominal cyst. A retrospective analysis of 41 cases of fetal abdominal cyst, which included ovarian cysts, choledochal cysts, intestinal duplication and mesenteric cysts, was performed. Imaging characteristics of various types of cysts were summarized, compared and discussed. Among 41 fetal abdominal cyst cases, there were 21 cases of ovarian cysts, 11 cases of bile duct cyst, six cases of intestinal duplication and three cases of mesenteric cyst. Each type of fetal cyst had its own distinctive characteristics on abdominal ultrasound examination. Ovarian cysts were located at one side of the bladder, round-shaped and observed in female fetuses; choledochal cysts were located in the hilar, were oblong- or oval-shaped and connected to the bile duct; intestinal duplication was located in the middle of abdomen, close to the intestine, and presented as an intestinal wall-like structure; mesenteric cysts were round-shaped with thin tensionless wall, presented with multiple chambers, and were easily deformable on compression. The findings of the present study demonstrated that a comprehensive analysis of the association between the cyst and its adjacent location, shape, wall thickness, motility and other aspects of dynamic changes via ultrasonography may provide a differential diagnosis of different types of fetal abdominal cysts.
\end{abstract}

\section{Introduction}

Fetal abdominal cysts are rare and few cases have been described in the literature. With recent advances in ultrasound techniques and increasing clinical experience, routine ultrasound screening has facilitated the early detection of fetal structural abnormalities (1-4). The abnormal cystic structures mainly originate from either gastrointestinal tract or genitourinary tract (5-9). However, since abdominal

Correspondence to: Dr Chang-Yu Tu, Department of Ultrasound, Women and Children's Hospital of Linyi, 1 Qinghe Road, Linyi, Shandong 276001, P.R. China

E-mail: changyutu2015@sina.com

Key words: fetus, ultrasound, ovarian cyst, choledochal cyst, intestinal duplication, mesenteric cyst cysts may be derived from different reproductive, urinary and digestive systems (6-9), it becomes more difficult to accurately determine the nature of cysts antenatally via ultrasound and predict the postnatal outcome.

In the present study, 41 cases of fetal abdominal cysts diagnosed by ultrasound were investigated to reveal the differences in ultrasound image features between the various different types of cyst and discuss how to differentiate from other diseases with similar characteristics. Further experience to differentiate between different types of cysts may assist in the clinical assessment of disease outcome and determine the choice of treatment

\section{Materials and methods}

Study subjects. A retrospective analysis of 41 cases with fetal abdominal cyst diagnosed by routine prenatal ultrasound examination between February 2005 and February 2015 at Women and Children's Hospital of Linyi (Linyi, China) was performed. In the present study, pregnant women were aged 21-39 years (mean, 24 years) with a range of 19-39 weeks of pregnancy. Among 41 pregnant women, 36 were primiparous whereas five were non-primiparous. When assessed during pregnancy, abdominal circumference and cervical length were coincident with gestational age, and no discomfort was observed in the pregnant women. The fetal abdominal cystic cases examined in the present study included ovarian cysts, choledochal cysts, intestinal duplication and mesenteric cysts. Other cysts, such as intraparenchymal cyst, cystic changes found in the urinary system and gastrointestinal obstruction were excluded.

Instruments and methods. Volusoiv E8,730 expert, Logiq E9 (both GE Healthcare Life Sciences, Logan, UT, USA) and Phillips iU22 color Doppler (Phillips Healthcare, DA Best, The Netherlands) ultrasonic diagnostic apparatus with a probe frequency of 3.0-5.0 HMz were used. Pregnant women were positioned in the supine position for a routine comprehensive fetal ultrasound. Ultrasound measurements included fetal biparietal diameter, head circumference, abdominal circumference, femur length, maximum anteroposterior diameter of amniotic fluid or amniotic fluid index, umbilical artery systolic/diastolic ratio, resistive index and pulsatility index. Subsequent multi-slice scanning of the fetal abdomen was performed, which included the fetal abdomen from the diaphragm to the pelvis level at each cross-section, sagittal section and oblique surface. In addition to scanning these 
sections, a continuous scan from one side of the fetal abdomen slowly sliding to the other side was performed, followed by another continuous scanning after the probe was rotated $90^{\circ}$.

\section{Results}

Patient characteristics. Of 41 cases with fetal abdominal cysts identified by regular ultrasound examination, ovarian cysts, choledochal cysts, duplication of small intestine and mesenteric cysts were observed in 21, 11, 6 and 3 cases respectively. All cases were confirmed by prenatal and postnatal ultrasound, surgery or abortion.

\section{Details of ultrasound manifestation}

Ovarian cysts. A representative ovarian cyst is presented in Fig. 1. Among 21 cases of fetal ovarian cysts which all appeared in female fetuses, 18 and 3 cases exhibited cysts detected in the lower and upper abdomen, respectively. In 20 cases of ovarian cysts found along the midline of the abdomen, 17 were detected at one side of the bladder and one at the midline. The majority of cases exhibited single cysts $(n=19)$, whereas two cases exhibited multiple cysts. There was no significant movement of cyst location in 15 cases whereas slight movement, predominantly up-and-down movement, was observed in six cases. The shortest and longest diameters of the ovarian cysts were 2.1 and $5.5 \mathrm{~cm}$ respectively. A total of 19 cases exhibited cysts that appeared as round-shaped, whereas two were oval-shaped, and all of them were regular in appearance. A total of 19 cases of cysts had a smooth contour, and only two did not. Cyst wall thickness $<0.3 \mathrm{~cm}$ was observed in 18 cases, whereas a thickness of $\geq 0.3 \mathrm{~cm}$ was detected in three cases. Good sound transmission was detected in nine cases, whereas two cases exhibited poor sound transmission, and all cases showed posterior echo enhancement. Upon repeated ultrasound examination, cysts were demonstrated to have reduced in size in 12 cases, had subsequently disappeared in four cases, exhibited no change in three cases, and had enlarged in two cases.

Choledochal cysts. A representative choledochal cyst is presented in Fig. 2. A total of 11 cases with choledochal cysts were identified. This type of cyst was seen at the hepatic hilar, behind the portal vein, in the right upper quadrant of the abdomen. All cases presented with single cysts. The smallest cyst size was $2.7 \times 2.1 \mathrm{~cm}$, whereas the largest one was $4.5 \times 3.2 \mathrm{~cm}$. Nine cysts were oval-shaped and two were round-shaped. All cysts were found connected to bile ducts, whereas only eight cases were clearly discernible and two cases were unclear. Furthermore, two cases presented with liver echo enhancement and three cases were complicated with intrahepatic bile duct cystic dilatation.

Duplication of small intestine. Of six cases that presented with duplication of the small intestine, five were observed at the lower abdomen and one on the right abdomen. Four cases were single cysts and two cases presented with multiple cysts. The majority of the cysts had a certain degree of motility. The smallest cyst size was $2.6 \times 1.8 \mathrm{~cm}$ and the largest one was $6.3 \times 2.0 \mathrm{~cm}$. Three cases were round-shaped and three cases were oblong- and oval-shaped. The wall of the cysts was relatively thicker compared with other types of cysts, which was closely associated with the digestive tract (Fig. 3). Three cases presented with intestinal wall-like structure and two cases exhibited cyst wall motility. The cyst showed a clear boundary in two cases, whereas the boundary was unclear in four cases. One case presented with a cyst near the intestinal dilatation.

Mesenteric cysts. Of three cases of mesenteric cysts, all were single cysts observed at the middle of the abdomen. Two cases were oval-shaped and one exhibited a irregular shape, which was possibly due to the effects of pressure. The cyst wall was relatively thin and was separated. Two cases demonstrated good sound transmission whereas the remaining cyst showed poor sound transmission. All cysts were movable and their shapes were altered when pressure was applied via the ultrasound probe.

\section{Discussion}

Etiology of fetal abdominal cysts. Ovarian cysts are the most common type of abdominal cysts in fetuses $(10,11)$; however, the etiology remains unclear. However, it has been postulated that the occurrence is associated with maternal and fetal gonadotropin levels. In particular, pregnant women who have preeclampsia, diabetes, maternal hypertension, maternal-fetal rhesus blood group incompatibility may have increased gonadotrophin levels, which may result in the formation of fetal follicular cysts

Intracapsular bleeding may occur in fetal ovarian cysts and hemorrhage may accompany a ruptured capsule. Torsion and necrosis of the cysts and ovary may occur and the larger cysts may compress or even cause obstruction of the gastrointestinal or urinary tract. While the majority of cases are benign and some cysts may disappear prior to or four months after birth, other cysts may continue to grow and present with torsion following birth (12). In the present study, there were two cases that exhibited cysts that increased in size after birth, among which one with torsion and necrosis required surgical resection.

A choledochal cyst is also known as congenital bile duct dilatation, primary bile duct dilatation or bile duct cystic dilatation. Fetal choledochal cysts are rare in the Chinese population, and neonatal incidence has been demonstrated to be $1 / 100,000-1 / 150,000$ (13). Although the etiology of the disease remains unclear, it has been suggested that pancreaticobiliary maljunction may cause pancreatic enzyme reflux into the biliary ducts and result in bile duct dilatation (14). Bile duct dysplasia was also suggested to be associated with developmental defects in periductal connective tissues, a thin bile duct wall, or proximal bile duct dilatation, which results from increased bile duct pressure induced by distal common bile duct obstruction. The lack of ganglion cells in the distal common bile duct wall may reduce the rhythmic movement of the bile duct, and the structural and functional abnormalities of its distal muscle may cause bile duct obstruction and subsequently bile duct dilation.

Intestinal duplication refers to the tubular hollow structure that appears in the small intestine mesentery. It can occur in any part of the intestine, although it is 


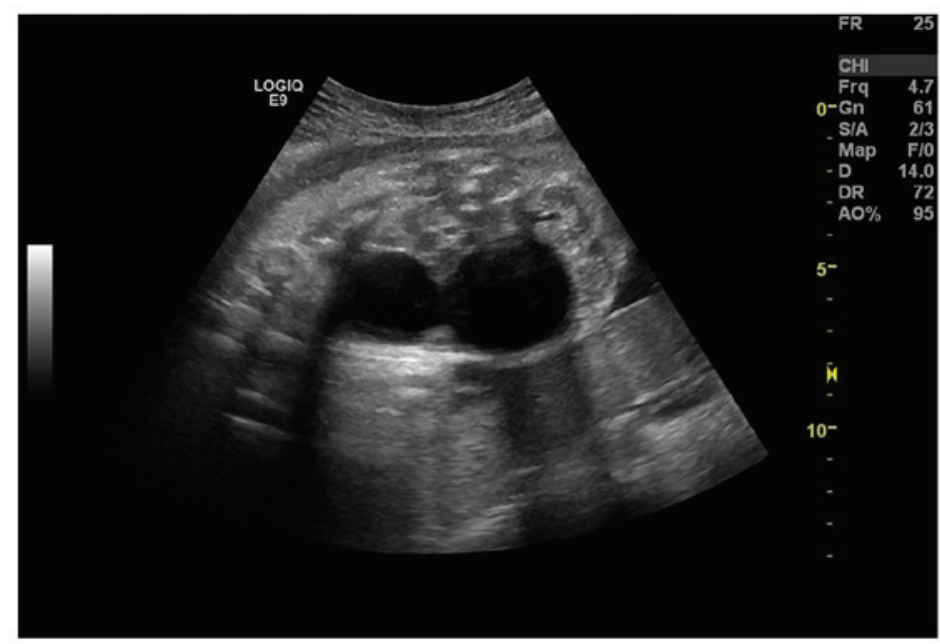

Figure 1. Fetal ovarian cysts. A cystic mass measuring $5.0 \times 4.5 \mathrm{~cm}$ was detected on the left side of the bladder.

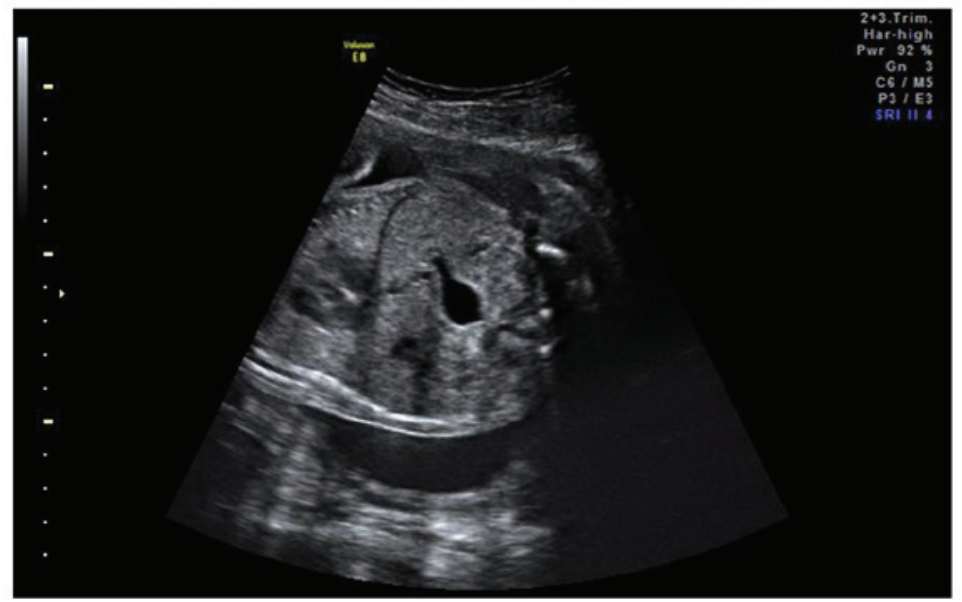

Figure 2. Fetal choledochal cyst. A fetal hilar cystic mass measuring $1.7 \times 1.3 \mathrm{~cm}$, complicated with the bile duct, was detected.

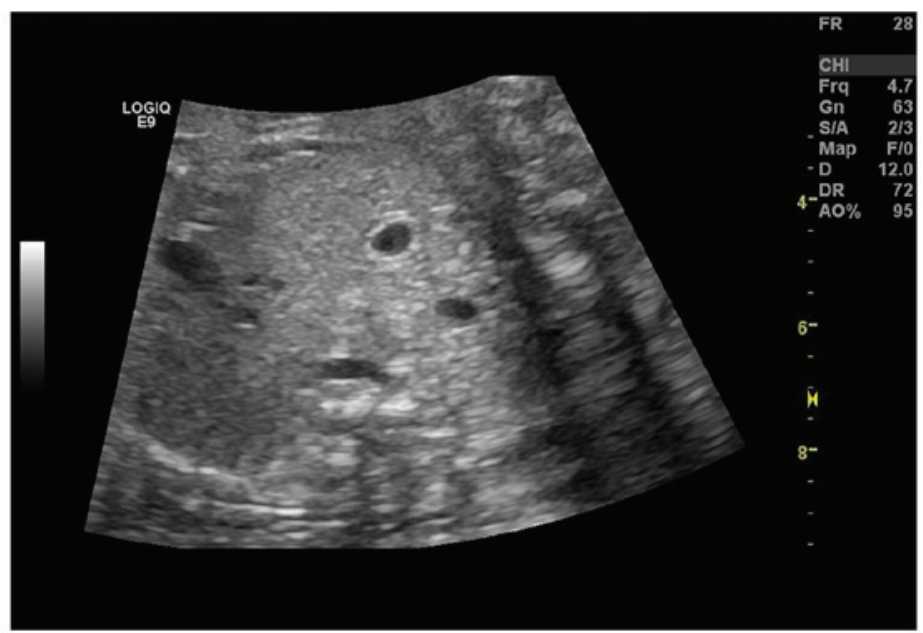

Figure 3. Fetal intestinal duplication. A cystic mass with a thick intestinal wall-like structure was detected in the left upper abdomen of the fetus.

predominantly observed in the ileum. Different theories have been proposed for the cause of the disease. One suggested that, during vacuole fusion in the lumenal space, duplication may form if the lumenal space is not fully integrated with the intestine (15). Another suggested that, during the early development of the embryonic digestive tract, the sac- and 
pouch-like diverticulum will gradually degenerate and disappear under normal circumstances, otherwise it will form cystic duplication (15). Other theories have suggested that intestinal duplication may be associated with developmental disorders of spinal cord and blood vessels (16).

Mesenteric cysts are generated from mesenteric lymphatic swelling and cystic changes. The cyst wall is composed of epithelial cells and connective tissues. Within the cyst, there is chylous fluid or a small amount of blood and cellulose, and it is sometimes referred to as a celiac mesenteric cyst. Formation of a mesenteric cyst may be due to congenital dysplasia of the lymphatic tissue, characterized by unusual tumor-like expansion of the lymphatic vessels. It has also been proposed that cyst formation is the result of lymph flow obstruction due to occlusion between the lymphatic and venous collaterals (17). Mesenteric cysts predominantly occur in the small bowel mesentery, which is near the mesenteric edge of the small intestine and a number have been known to occur beyond the mesocolon or retroperitoneal colon (18).

Ultrasound imaging features of fetal abdominal cysts. With a certain regularity of ultrasound images, fetal abdominal cysts were observed in female fetuses, and they were predominantly found in the lower abdomen or pelvis, with a few in the upper abdomen close to one side of the bladder. The majority of the cases presented with a singular cysts, and only a few individual cases exhibited multiple cysts. The majority of the cysts were able to move slightly up and down and their diameters were measured at $\sim 4.0 \mathrm{~cm}$, with only a few $>6.0 \mathrm{~cm}$. The shape of the cysts was regular, and they were predominantly round- or oval-shaped. The cysts wall was generally thin with a smooth contour. Regular ultrasound scanning revealed that the cysts decreased in size or disappeared over time; however, some cases did not exhibit any significant change in size, whereas others increased in size.

Choledochal cysts were found in the right upper quadrant behind the portal vein and were predominantly oval-shaped. This type of cysts was characterized by its connection with the bile ducts and gallbladder, which was observed in 11 cases in the present study. Intestinal duplication cysts presented as a round- or oval-shaped mass, and a few of them were oblong- or tubular-shaped. The cyst wall was thicker and exhibited an intestinal wall-like structure that was closely associated with the digestive tract. This type of cyst was movable to a certain degree when manipulated by the ultrasound probe. In addition, it shared the same blood vessels with the accompanying intestine, and in some individual cases, the cyst compressed the adjacent intestine resulting in intestinal obstruction $(19,20)$. In the present study, mesenteric cysts were solitary, and observed to be unilocular or multilocular on ultrasound. These cysts tended to be roundor oval-shaped with thin walls that lacked tension. Those close to the intestine may form dumbbell cysts (21), and were movable or deformable when manipulated by the ultrasound probe.

Comprehensive analysis of the fetal abdominal cyst. Fetal abdominal cyst may be derived from different organ systems. Since most of the characteristics are consistent between the different types of cysts on ultrasound examination, accurate ultrasound diagnosis remains difficult. Therefore, it is important to identify the cystic characteristics on ultrasound examination and integrate these with clinical observations in order to achieve a correct diagnosis. To accurately identify the type of fetal abdominal cyst, the following aspects should be considered (22):

Cyst location and adjacent structures. Ovarian cysts are commonly found at one side of the bladder in the lower abdomen and can be found at the middle of abdomen; however, they are rarely observed in the upper abdomen. Choledochal cysts are typically found at the right upper quadrant of the abdomen, close to the liver, with its rear part near the portal vein. Since this type of cyst is fixed in position, it can be easily differentiated from others types of cyst. Although the locations of intestinal duplication and mesenteric cyst remain relatively variable, they are commonly found at the middle of the abdomen. Intestinal duplication cysts can be found along the small intestine and are closely associated with the adjacent bowel.

Cyst morphology and tension. Ovarian cysts are predominantly round- and oval-shaped with high tension; choledochal cyst are non-circular and are typically ovaland oblong-shaped with low tension; intestinal duplication cysts may be round, oval, oblong, or tubular in shape with a certain tension; and mesenteric cysts are round-, oval-, or dumbbell-shaped with low or no tension, thus they are easily deformed under pressure.

Cyst wall thickness. The ovarian cyst wall is typically thinner than that of other types of cyst, with smooth contours; the choledochal cyst wall is slightly thicker with discontinuous wall contours that can be seen in connection with the bile duct or gallbladder; the wall of an intestinal duplication cyst is thicker with smooth contours and appears as an intestinal wall-like structure, running in parallel with the major intestinal wall, with careful observation; and the mesenteric cyst wall is thin, with separate capsules observed seen within the cyst.

Cyst motility. Ovarian cyst exhibit a certain degree of motility; choledochal cysts are relatively fixed in position; mesenteric and intestinal duplication cysts both have a greater degree of motility when manipulated by the ultrasound probe; peristalsis is occasionally observed in intestinal duplication cysts (23).

Follow-up observation. Larger alterations in size are observed in ovarian cysts and the majority reduce in size or disappear. However, choledochal cysts become larger in size on follow-up observation, and intestinal duplication and mesenteric cysts typically do not change in size at all during follow-up.

\section{Ultrasound diagnosis of fetal abdominal cyst}

Ovarian cysts. Ovarian cyst can be differentiated from ureteral dilatation, which is seen as a tubular structure visualized behind the bladder on ultrasound that is typically accompanied by an ipsilateral hydronephrosis. Ovarian cysts can be easily mistaken as the bladder when there is no urine in the bladder. However, ovarian cysts are often located at one side of the abdomen midline, whereas the bladder is located along the lower abdomen midline near to the anterior abdominal wall (24). On Color Doppler ultrasonography, 
umbilical artery blood flow is observed at both sides of the bladder with alterations in size from dynamic observation. When compared with ovarian cysts, urachal cysts are found in the superficial area close to the anterior abdominal wall in the middle of the abdomen. Anal atresia, during which bowel dilatation in the lower pelvis shows the 'double leaf sign' image, which is different from the circular structure of ovarian cyst (25).

Choledochal cysts. Distinct from the larger size of choledochal cysts, which become bigger in size with increasing gestational age and are accompanied by normal gallbladder, cystic biliary atresia tends to be smaller with no significant alterations in cystic size. Furthermore, the gallbladder is typically not visualized or is small. When compared with choledochal cysts, the image exhibits a double-bubble sign that is observed during duodenal obstruction, accompanied by the enlarged stomach bubble. Notably, the enlarged duodenum is not connected with the bile duct.

Intestinal duplication. Intestinal duplication can be differentiated from small bowel obstruction where the resulting enlarged intestine is displayed as echo-free and multiple stacked or a honeycomb-shaped tube $(26,27)$.

Mesenteric cysts. Distinct from omental cysts, mesenteric cysts are small and distributed in the intestine. On occasions, the small intestine can be observed between the cyst and the anterior abdominal wall and it may compress the other intestine. Omental cysts are typically found in the upper abdomen, closer to the anterior abdominal wall, and are larger in size compared to mesenteric cysts. Mesenteric cysts are also distinguishable from cystic teratoma, which have a thicker cyst wall and calcification on image analysis. In addition, cystic teratoma are typically associated with poor sound transmission and an uneven echo. Sometimes, hyperechoic floating fat balls or globules can be observed inside the teratoma (28).

In conclusion, there are various different types of fetal abdominal cyst that are distinguished by the site of occurrence and have a differential impact on fetal development. Prenatal ultrasound is important to identify the cause and nature of the cyst, assist in the assessment of disease outcome and determine the choice of treatment. An appropriate ultrasound examination with comprehensive analysis and careful judgement would therefore directly affect the accuracy of diagnosis and proper treatment of the fetal abdominal cyst.

\section{References}

1. Snijders RM, Noble P, Sebire N, Souka A and Nicolaides KH: UK multicentre project on assessment of risk of trisomy 21 by maternal age and fetal nuchal-translucency thickness at 10-14 weeks of gestation. Fetal medicine foundation first trimester screening group. Lancet 352: 343-346, 1998.

2. Nicolaides KH: Nuchal translucency and other first-trimester sonographic markers of chromosomal abnormalities. Am J Obstet Gynecol 191: 45-67, 2004.

3. Souka AP, Pilalis A, Kavalakis Y, Kosmas Y, Antsaklis P and Antsaklis A: Assessment of fetal anatomy at the 11-14-week ultrasound examination. Ultrasound Obstet Gynecol 24 730-734, 2004.
4. Malone FD, Canick JA, Ball RH, Nyberg DA, Comstock CH, Bukowski R, Berkowitz RL, Gross SJ, Dugoff L, Craigo SD, et al: First-trimester or second-trimester screening, or both, for Down's syndrome. N Engl J Med 353: 2001-2011, 2005.

5. Gabrielli S, Rizzo N and Reece EA: Gastrointestinal and genitourinary anomalies. In: Clinical Obstetrics. The Fetus \& Mother (3rdedn). Reece EA and Hobbins JC (eds). Blackwell Publishing, Malden, MA, pp377-400, 2007.

6. Hyett J: Intra-abdominal masses: Prenatal differential diagnosis and management. Prenat Diagn 28: 645-655, 2008.

7. McEwing R, Hayward C and Furness M: Foetal cystic abdominal masses. Australas Radiol 47: 101-110, 2003.

8. Nyberg DA and Neilson IR: Abdomen and gastrointestinal tract. In Diagnostic Imaging of Fetal Anomalies, Nyberg DA, McGahan JP, Pretorius DH, Pilu G (eds) Lippincott Williams \& Wilkins: Philadelphia, PA, pp547-602, 2003.

9. Hill LM: Ultrasound of fetal gastrointestinal tract. In Ultrasonography in Obstetrics and Gynecology (4th edn), Callen PW (ed.) W. B. Saunders: Philadelphia, PA, pp457-487, 2000.

10. Chang H, Li Y, Liu Y, Cao H, Zhang F and Pan Q: Ultrasound diagnosis of fetal ovarian cysts. Zhong Hua Chao Sheng Ying Xiang Xue Za Zhi 11: 253-254, 2002 (In Chinese).

11. Feng W, Cui G and Fu T: Advances in diagnosis and treatment of fetal ovarian cysts. Contemp Med 18: 274-275, 2012.

12. deSa DJ: Follicular ovarian cysts in stillbirths and neonates Arch Dis Child 50: 45-50, 1975.

13. Hu J, Liu Q, Wu Y, Zhou Y, Wang J, and Pan W: Clinical analysis of prenatal diagnosis of choledochal cyst in 37 infants. J Clin Pediatr 3: 858-861, 2013.

14. Jensen KK and Sohaey R: Antenatal sonographic diagnosis of choledochal cyst: Case report and imaging review. J Clin Ultrasound 43: 581-583, 2015.

15. Stern LE and Warner BW: Gastrointestinal duplications. Semin Pediatr Surg 9: 135-140, 2000.

16. Zahir I, Yusuf S, Zada F, Asif M, Akhtar N, Abbasi MZ: Duplication Cyst in a New Born. Int J Path 8: 84-86, 2010

17. Pilu G and Nicolaides KH: Gastrointestinal tract. In Diagnosis of fetal abnormalities: The 18-23-week scan, Nicolaides KH (ed) Parthenon Publishing Group: London, pp74, 1999.

18. Kurtz RJ, Heimann TM, Holt J and Beck AR: Mesenteric and retroperitoneal cysts. Ann Surg 203: 109-112, 1986.

19. Lan Y, Xue X, Wang S and Liu N: Ultrasound diagnosis of fetal intestinal duplication: a case report. Zhong Hua Chao Sheng Ying Xiang Xue Za Zhi 15: 119, 2006 (In Chinese).

20. Pu H: Ultrasound diagnosis of fetal cecal duplication: A case report. Zhong Guo You Sheng Yu Yi Chuan Za Zhi She 19: 112, 2011. (In Chinese).

21. Ge Q, Li M, Li Y, Lin L and Huan F. Fetal abdominal cystic mass of prenatal ultrasound diagnosis and differential diagnosis. Modern Instruments \& Medical Treatment 21: 10-12, 2015 (In Chinese)

22. Tu C: Obstetric ultrasound measurements and diagnostics (1st edn). Vol. 1: Shandong Science and Technology Press, Jinan, 2014.

23. Wang L, Ma X, Pan Y, Zhang H, Zhang J and An S: The antenatal sonographic diagnosis and differential diagnosis of fetal abdominal cysts. Chin J Clinicians (Electronic Edition) 7: 87-90, 2013 (In Chinese)

24. Carlson DH and Griscom NT: Ovarian cysts in the newborn. Am J Roentgenol Radium Ther Nucl Med 116: 664-672, 1972.

25. Cao H and Deng X: Study on the correlation between fetal bowel dilatation and pregnancy outcomes. Chin J Med Ultrasound 7: 577-581, 2014. (Electronic Edition).

26. Yang $\mathrm{J}$ and $\mathrm{Ma} \mathrm{D}$ : Clinical and imaging diagnosis of intestinal duplication in children. J Appl Clin Pediatr 23: 545-547, 2008. (In Chinese)

27. Chen X, Huang F and Pan Y: Ultrasound diagnosis of intestinal duplication. Chin J Ultrasound Diagn 7: 108-110, 2006. (In Chinese)

28. Mahomedy S, Bayat MR and Seedat M: Meat balls: A pathognomonic ultrasound and computed tomography finding in mature cystic teratoma. Australas Radiol 51 (Suppl): B281-B283, 2007. 